\title{
Brownian-Motion Model for Parametric Correlations in the Spectra of Disordered Metals
}

\author{
C. W. J. Beenakker \\ Instituut-Lorentz, University of Leiden, P.O. Box 9506, 2300 RA Leiden, The Netherlands
}

(Received 11 March 1993)

\begin{abstract}
We study the response to an external perturbation of the energy levels of a disordered metallic particle, by means of the Brownian-motion model introduced by Dyson in the theory of random matrices, and reproduce the results of a recent microscopic theory [A. Szafer and B. L. Altshuler, Phys. Rev. Lett. 70, 587 (1993)]. This establishes the validity of Dyson's basic assumption, that parametric correlations in the energy spectrum are dominated by "level repulsion," and therefore solely dependent on the symmetry of the Hamiltonian.
\end{abstract}

PACS numbers: $73.20 . D x, 05.40 .+\mathrm{j}, 05.45 .+\mathrm{b}, 71.25 .-\mathrm{s}$

In 1965, Gorkov and Eliashberg [1] proposed to describe the electronic excitation spectrum of small metallic particles in terms of the Wigner-Dyson theory for the statistical properties of the eigenvalues of random Hermitian matrices. The basic assumption of this randommatrix theory (RMT) is that the spectral correlations are dominated by level repulsion [2]. Level repulsion is a direct consequence of the transformation from the space of $N \times N$ matrices $\mathcal{H}$ to the smaller space of $N$ eigenvalues $E_{i}$. Level repulsion is "universal" in the sense that it is fully determined by the symmetry class of the Hamiltonian ensemble. There exist just three symmetry classes, characterized by the index $\beta: \beta=1$ in zero magnetic field (orthogonal ensemble), $\beta=2$ in nonzero field (unitary ensemble), and $\beta=4$ for strong spin-orbit scattering in zero magnetic field (symplectic ensemble) $[3,4]$.

It was not until twenty years later that the basic assumption of RMT was justified by a microscopic theory, by Efetov [5] and by Altshuler and Shklovskiu [6]. These authors showed that the correlation function of pairs of energy levels agrees with RMT for level separations $\delta E$ up to the Thouless energy $E_{c} \simeq \hbar v_{F} l / L^{2}$ (where $L$ is the diameter of the particle, $v_{F}$ the Fermi velocity, and the mean free path $l$ is $\ll L$ ). In the energy range $\Delta \ll \delta E \ll E_{c}$ (with $\Delta$ the mean level spacing) the pair correlation function is universal, i.e., independent of the particle size or the degree of disorder. Only the symmetry index $\beta$ remains as a relevant parameter.

In a recent publication [7], Szafer and Altshuler have used the diagrammatic perturbation theory of Ref. [6] to study the response of the energy levels to an external perturbation. They considered a metallic particle with the topology of a ring, enclosing a magnetic flux $\phi$ (measured in units of $h / e)$. The energy levels $E_{i}(\phi)$ depend parametrically on $\phi$. Their dispersion is characterized by the "current density"

$$
j(E, \phi)=\sum_{i=1}^{N} \delta\left(E-E_{i}(\phi)\right) \frac{d}{d \phi} E_{i}(\phi) .
$$

Szafer and Altshuler found that the correlation function

$$
C(\delta E, \delta \phi)=\overline{j(E, \phi) j(E+\delta E, \phi+\delta \phi)}
$$

becomes universal for $\delta E=0$ and $\left(\Delta / E_{c}\right)^{1 / 2} \ll \delta \phi \ll 1$ :

$$
C(0, X)=-2 / \pi^{2} \beta X^{2},
$$

with $\beta=2$ and $X=\delta \phi$.

Equation (3) was proven for the case that the randomness in the energy spectrum is due to scattering by randomly located impurities. [The overline in Eq. (2) then denotes an average over the impurity configurations.] Numerical simulations indicated that it applies generically to chaotic systems, even if there is no disorder and all randomness comes from scattering at irregularly shaped boundaries [7]. (The average in that case is taken over $E$ and $\phi$.) Further work on disordered systems by Simons and Altshuler [8], based on the nonperturbative supersymmetry formalism of Ref. [5], has shown that Eq. (3) with $\beta=1$ and $X=\delta U$ applies if the external perturbation is a spatially fluctuating electrostatic potential $U_{s}(\mathbf{r})$. The correlator (3) thus provides a universal quantum mechanical characterization of the response of a chaotic system to an external magnetic or electric field, including such diverse applications as nuclear deformation, chaotic billiards, persistent currents in an Aharonov-Bohm ring, and dispersion relations of complex crystalline lattices $[7,8]$.

Such universality suggests that it should be possible to derive Eq. (3) from the basic assumption of RMT, that spectral correlations are due to level repulsion and therefore fully determined by symmetry. The purpose of this paper is to show how this can be achieved.

The starting point of our analysis is Dyson's Brownianmotion model [9] for the evolution of an ensemble of $N \times N$ random matrices as a function of an external parameter $\tau$. Dyson's idea was to regard $\tau$ as a fictitious "time," and to model the $\tau$ dependence of the distribution of energy levels $P\left(\left\{E_{n}\right\}, \tau\right)$ by the one-dimensional Brownian motion of $N$ classical particles at positions $E_{i}(\tau)$, in a fictitious viscous fluid with friction coefficient $\gamma$ and temperature $\beta^{-1}$. Level repulsion is accounted for 
by the interaction potential $-\ln \left|E-E^{\prime}\right|$ between particles at $E$ and $E^{\prime}$. The particles move in a confining potential $V(E)$, which is determined by the density of states (assumed to be independent of $\tau$ ).

The fictitious time $\tau$ needs still to be related to the perturbation parameter $X$ in the Hamiltonian $\mathcal{H}(X)$ of the physical system one is modeling. Let $\tau=0$ coincide with $X=0$, so that

$$
P\left(\left\{E_{n}\right\}, 0\right)=\prod_{i} \delta\left(E_{i}-E_{i}^{0}\right),
$$

with $E_{i}^{0}$ the eigenvalues of $\mathcal{H}(0)$. For $\tau>0$ we then identify

$$
\tau=X^{2}
$$

This is the simplest relation between $\tau$ and $X$ which is consistent with the initial rate of change of the energy levels: On the one hand, $\left[E_{i}(X)-E_{i}^{0}\right]^{2}=X^{2}\left(d E_{i} / d X\right)^{2}$ is of order $X^{2}$ for small $X$, while on the other hand the ensemble average $\left\langle\left[E_{i}(\tau)-E_{i}^{0}\right]^{2}\right\rangle=2 \tau / \beta \gamma$ is of order $\tau$ for small $\tau$ [9]. For later use we also note the relation

$$
2 / \beta \gamma=\overline{\left(d E_{i} / d X\right)^{2}}
$$

between the friction coefficient and the mean-square rate of change of the energy levels, which is implied by the identification (5).

With these definitions, $P\left(\left\{E_{n}\right\}, \tau\right)$ evolves according to the $N$-dimensional diffusion equation [9]

$$
\begin{gathered}
\frac{\partial P}{\partial \tau}=\sum_{i=1}^{N} \frac{\partial}{\partial E_{i}} \gamma^{-1}\left(P \frac{\partial W}{\partial E_{i}}+\beta^{-1} \frac{\partial P}{\partial E_{i}}\right), \\
W\left(\left\{E_{n}\right\}\right)=-\sum_{i<j} \ln \left|E_{i}-E_{j}\right|+\sum_{i} V\left(E_{i}\right) .
\end{gathered}
$$

Equation (7) has the $\tau \rightarrow \infty$ ("equilibrium") solution

$$
P_{\text {eq }}\left(\left\{E_{n}\right\}\right)=Z^{-1} e^{-\beta W},
$$

where $Z$ is such that $P_{\text {eq }}$ is normalized to unity. Equation $(8)$, for $\beta=1,2$, and 4 , is the eigenvalue distribution in the orthogonal, unitary, and symplectic ensemble [4].

Equation (7) is the simplest description of the Brownian motion of the energy levels which is consistent with the equilibrium distribution (8). It is not the most general description: (1) One could include the "velocities" $d E_{n} / d \tau$ as independent stochastic variables, and work with a $2 \mathrm{~N}$-dimensional diffusion equation. Instead, in the Brownian-motion model the finite relaxation time $\tau_{c}$ (specified below) of the velocities is ignored. This restricts the applicability to parameter ranges ("time scales") greater than $\tau_{c}$. (2) One could let $\gamma$ be a matrix function $\gamma_{i j}\left(\left\{E_{n}\right\}\right)$ of the configuration of energy levels. Such a configuration dependence (known in fluids as hydrodynamic interaction) would be an additional source of correlations, which is ignored. That is the basic assumption of Dyson's Brownian-motion model, that the spectral correlations are dominated by the fundamental geometric effect of level repulsion. The Brownian-motion model is known to provide a rigorous description of the transition between random-matrix ensembles of different symmetry [10]. However, there exists no derivation of Eq. (7) from a microscopic Hamiltonian. Here we apply the Brownian-motion model to fluctuations around equilibrium in the random-matrix ensembles (8), and show that there is a complete agreement with the microscopic theory for disordered metals by Szafer and Altshuler [7].

The first step in the analysis is to reduce Eq. (7) to an evolution equation for the average density of eigenvalues

$$
\rho(E, \tau)=\int_{-\infty}^{\infty} d E_{1} \cdots d E_{N} P\left(\left\{E_{n}\right\}, \tau\right) \sum_{i=1}^{N} \delta\left(E-E_{i}\right)
$$

This problem was solved by Dyson [9] in the limit $N \rightarrow$ $\infty$, with the result

$$
\gamma \frac{\partial}{\partial \tau} \rho(E, \tau)=\frac{\partial}{\partial E}\left[\rho(E, \tau) \frac{\partial}{\partial E}\left(V(E)-\int_{-\infty}^{\infty} d E^{\prime} \rho\left(E^{\prime}, \tau\right) \ln \left|E-E^{\prime}\right|\right)\right] .
$$

Corrections to Eq. (10) are smaller by an order $N^{-1} \ln N$. To the same order, $\rho_{\mathrm{eq}}(E)$ satisfies [9]

$$
\frac{\partial}{\partial E}\left(V(E)-\int_{-\infty}^{\infty} d E^{\prime} \rho_{\mathrm{eq}}\left(E^{\prime}\right) \ln \left|E-E^{\prime}\right|\right)=0 .
$$

The next step is to reduce Eq. (10) to a diffusion equation by linearizing $\rho$ around $\rho_{\text {eq }}$. We write $\rho(E, \tau)=\rho_{\text {eq }}(E)+$ $\delta \rho(E, \tau)$ and find, to first order in $\delta \rho$,

$$
\begin{gathered}
\frac{\partial}{\partial \tau} \delta \rho(E, \tau)=\frac{\partial}{\partial E} \int_{-\infty}^{\infty} d E^{\prime} D\left(E, E^{\prime}\right) \frac{\partial}{\partial E^{\prime}} \delta \rho\left(E^{\prime}, \tau\right) \\
D\left(E, E^{\prime}\right)=-\gamma^{-1} \rho_{\mathrm{eq}}(E) \ln \left|E-E^{\prime}\right|
\end{gathered}
$$

Equation (12) has the form of a nonlocal diffusion equa- tion, with diffusion kernel $D\left(E, E^{\prime}\right)$.

To proceed we assume a constant density of states over the energy range of interest, $\rho_{\text {eq }}(E) \equiv \rho_{0} \equiv 1 / \Delta$. The diffusion kernel then becomes translationally invariant, $D\left(E, E^{\prime}\right) \equiv D\left(E-E^{\prime}\right)$, with Fourier transform

$$
D(k) \equiv \int_{-\infty}^{\infty} d E e^{i k E} D(E)=\frac{\rho_{0} \pi}{\gamma|k|}
$$

Equation (12) becomes an ordinary differential equation in $k$ space, with solution

$$
\delta \rho(k, \tau)=\delta \rho(k, 0) \exp \left[-k^{2} D(k) \tau\right]
$$

In view of Eq. (4), the initial condition on the eigenvalue density is 


$$
\rho(E, 0)=\sum_{i} \delta\left(E-E_{i}^{0}\right) .
$$

We define the equilibrium average $\langle f\rangle_{\text {eq }}$ of an arbitrary function $f\left(\left\{E_{n}^{0}\right\}\right)$ of the initial configuration by

$$
\langle f\rangle_{\mathrm{eq}}=\int_{-\infty}^{\infty} d E_{1}^{0} \cdots \int_{-\infty}^{\infty} d E_{N}^{0} P_{\mathrm{eq}}\left(\left\{E_{n}^{0}\right\}\right) f\left(\left\{E_{n}^{0}\right\}\right) .
$$

The density-density correlation function $S\left(E, E^{\prime}, \tau\right)$ is defined by

$$
\begin{aligned}
S\left(E, E^{\prime}, \tau\right) & =\left\langle\rho(E, \tau) \rho\left(E^{\prime}, 0\right)\right\rangle_{\mathrm{eq}} \\
& =\left\langle\delta \rho(E, \tau) \delta \rho\left(E^{\prime}, 0\right)\right\rangle_{\mathrm{eq}}+\rho_{\mathrm{eq}}(E) \rho_{\mathrm{eq}}\left(E^{\prime}\right),
\end{aligned}
$$

where we have used that $\langle\rho(E, \tau)\rangle_{\text {eq }} \equiv \rho_{\text {eq }}(E)$. The pair correlation function $K\left(E, E^{\prime}\right)$ is related to $S\left(E, E^{\prime}, 0\right)$ by

$$
K\left(E, E^{\prime}\right)=\rho_{\mathrm{eq}}(E) \rho_{\mathrm{eq}}\left(E^{\prime}\right)-S\left(E, E^{\prime}, 0\right) .
$$

In the case of a constant density of states, the correlation functions $S\left(E, E^{\prime}, \tau\right) \equiv S\left(E-E^{\prime}, \tau\right)$ and $K\left(E, E^{\prime}\right) \equiv$ $K\left(E-E^{\prime}\right)$ are translationally invariant, with Fourier transforms $S(k, \tau)$ and $K(k)$. According to Eqs. (14), $(17)$, and (18), we have

$$
S(k, \tau)=2 \pi \rho_{0}^{2} \delta(k)-K(k) \exp \left[-k^{2} D(k) \tau\right] .
$$

The function $K(k)$ is known from RMT [4]. In the limit $N \rightarrow \infty$, one has asymptotically

$$
K(k)=-|k| / \pi \beta,
$$

independent of $V(E)$ [11]. Combining Eqs. (13), (19), and $(20)$, we conclude that

$$
S(k, \tau)=2 \pi \rho_{0}^{2} \delta(k)+\frac{|k|}{\pi \beta} \exp \left(-\pi \rho_{0}|k| \tau / \gamma\right) .
$$

We are now ready to make the connection with the universal correlator (3). We define the correlation functions

$$
\begin{gathered}
S\left(E, X, E^{\prime}, X^{\prime}\right)=\sum_{i, j} \overline{\delta\left(E-E_{i}(X)\right) \delta\left(E^{\prime}-E_{j}\left(X^{\prime}\right)\right)} \\
C\left(E, X, E^{\prime}, X^{\prime}\right)=\sum_{i, j} \overline{\dot{E}_{i}(X) \dot{E_{j}}\left(X^{\prime}\right) \delta\left(E-E_{i}(X)\right) \delta\left(E^{\prime}-E_{j}\left(X^{\prime}\right)\right)},
\end{gathered}
$$

where $\dot{E}_{i} \equiv d E_{i} / d X$. By definition,

$$
\frac{\partial^{2}}{\partial E \partial E^{\prime}} C\left(E, X, E^{\prime}, X^{\prime}\right)=\frac{\partial^{2}}{\partial X \partial X^{\prime}} S\left(E, X, E^{\prime}, X^{\prime}\right) .
$$

Translational invariance reduces $C$ and $S$ to functions $C\left(E-E^{\prime}, X-X^{\prime}\right)$ and $S\left(E-E^{\prime}, X-X^{\prime}\right)$ of the energy and parameter increments only. Relation (24) becomes, upon Fourier transformation of the energy variable,

$$
C(k, X)=-\frac{1}{k^{2}} \frac{\partial^{2}}{\partial X^{2}} S(k, X) .
$$

The correlation functions $S(k, X)$ and $C(k, X)$ follow from Eqs. (5), (21), and (25),

$$
\begin{aligned}
& S(k, X)=2 \pi \rho_{0}^{2} \delta(k)+\frac{|k|}{\pi \beta} \exp \left(-\xi^{2}|k|\right), \\
& C(k, X)=\frac{2 \rho_{0}}{\beta \gamma}\left(1-2 \xi^{2}|k|\right) \exp \left(-\xi^{2}|k|\right),
\end{aligned}
$$

where we have abbreviated $\xi \equiv X\left(\pi \rho_{0} / \gamma\right)^{1 / 2}$. The $E$ space correlation functions become

$$
\begin{aligned}
& S(E, X)=\rho_{0}^{2}+\frac{1}{2 \pi^{2} \beta} \frac{\partial^{2}}{\partial E^{2}} \ln \left(\xi^{4}+E^{2}\right), \\
& C(E, X)=\frac{1}{2 \pi^{2} \beta} \frac{\partial^{2}}{\partial X^{2}} \ln \left(\xi^{4}+E^{2}\right) .
\end{aligned}
$$

In the limit $E \rightarrow 0$, Eq. (29) reduces to

$$
\begin{aligned}
C(0, X) & =\frac{2}{\pi^{2} \beta} \frac{\partial^{2}}{\partial X^{2}} \ln |X| \\
& =-\frac{2}{\pi^{2} \beta X^{2}}, \text { if } X \neq 0,
\end{aligned}
$$

independent of the microscopic parameters $\rho_{0}$ and $\gamma$. Equation (30), obtained here from random-matrix theory, is precisely the universal correlator (3) which Szafer and Altshuler [7] derived from diagrammatic perturbation theory. This is the fundamental result of Dyson's Brownian-motion model, which we now discuss in some more detail.

At $X=0, C(0, X)$ has an integrable singularity consisting of a positive peak such that the integral over all $X$ vanishes. This is a special case of the general sum rule $\int_{0}^{\infty} d X C(E, X)=0$, which follows from Eq. (24). The peak of positive correlation has infinitesimal width in the Brownian-motion model. In reality the peak has a finite width $X_{c} \equiv \sqrt{\tau_{c}}$ and a finite height $C(0,0) \simeq \rho_{0}^{2} \overline{\dot{E}_{i}^{2}} \equiv C_{0}$. The width and height are related by

$$
C_{0} X_{c} \simeq-\int_{X_{c}}^{\infty} C(0, X) d X \simeq 1 / X_{c} \Rightarrow C_{0} \simeq 1 / X_{c}^{2} .
$$

In terms of the generalized Thouless energy [8]

$$
\mathcal{E}_{c} \equiv C_{0} / \rho_{0} \equiv \rho_{0} \overline{\dot{E}_{i}^{2}}
$$

we obtain the estimate $X_{c} \simeq\left(\rho_{0} \mathcal{E}_{c}\right)^{-1 / 2}$. In Ref. [7] the parameter $X$ is the magnetic flux increment in units of 
$h / e$. Then $\mathcal{E}_{c}$ is the conventional Thouless energy $E_{c}$ [12], related to the conductance $g$ (in units of $e^{2} / h$ ) by $g \simeq \rho_{0} E_{c}$. The Aharonov-Bohm periodicity implies in this case the additional restriction $X \ll 1$ to Eq. (30) (which is compatible with the condition $X \gg X_{c}$ because $X_{c} \simeq g^{-1 / 2} \ll 1$ in the metallic regime).

We have shown that the $E \rightarrow 0$ limit of $C(E, X)$ obtained from RMT agrees with the microscopic theory. What about nonzero energy differences? This is most easily discussed in terms of the density-density correlation function $S(E, X)$, to which $C(E, X)$ is directly related via Eq. (25). Using Eqs. (6) and (31) we find that the result (28) can be rewritten identically as

$$
S(E, X)=\rho_{0}^{2}+\frac{1}{\pi^{2} \beta} \operatorname{Re}\left(i E+\frac{1}{2} \pi \beta \mathcal{E}_{c} X^{2}\right)^{-2},
$$

which coincides precisely with the result of diagrammatic perturbation theory $[7,8]$.

This establishes the validity of Dyson's Brownianmotion model for parametric correlations in the spectra of disordered metals, and places it on the same footing as the Wigner-Dyson theory for parameter-independent correlations.

We conclude by identifying some directions for future research. The restriction $X \gg X_{c}$ (or, equivalently, $\tau \gg \tau_{c}$ ) of the Brownian-motion model might be relaxed by introducing the derivatives $d E_{2} / d \tau$ as independent stochastic variables in a $2 N$-dimensional diffusion equation. It would be interesting to see if one could in this way reproduce the small- $X$ results of the microscopic theory [8]. The Brownian-motion model might also be extended to a parameter vector $X_{\mu}(\mu=1,2, \ldots, d)$, relevant for a statistical description of the dispersion relation of a $d$ dimensional crystalline lattice [8]. The Brownian motion would then take place in a fictitious world with multiple temporal dimensions $\tau_{\mu}$. An altogether different line of research would be to apply the Brownian-motion model to the response of the transmission eigenvalues $T_{i}$ to an external perturbation. The analog of level repulsion for the transmission eigenvalues is known [13], and leads to a pair correlation function $K\left(T, T^{\prime}\right)$ which differs from $\mathrm{Eq}$. (20) for $K\left(E, E^{\prime}\right)$ but has the same universal $\beta$ depen- dence [11]. This suggests that the analog of the universal correlator (3) exists as well for the transmission eigenvalues. Finally, the results of this paper and of Ref. [14] taken together imply a correspondence between Dyson's Brownian-motion model and the Sutherland Hamiltonian [15], which remains to be fully understood.

My interest in this topic was raised by a seminar of B. L. Altshuler at the Mittag-Leffler Institute (Djursholm, Sweden), the hospitality of which is gratefully acknowledged. I thank B. L. Altshuler and B. D. Simons for sharing their unpublished results with me, and for valuable correspondence. This work was supported in part by the Dutch Science Foundation NWO/FOM.

[1] L. P. Gorkov and G. M. Eliashberg, Zh. Eksp. Teor. Fiz. 48, 1407 (1965) [Sov. Phys. JETP 21, 940 (1965)].

[2] E. P. Wigner, SIAM Rev. 9, 1 (1967).

[3] F. J. Dyson, J. Math. Phys. 3, 1199 (1962).

[4] M. L. Mehta, Random Matrices (Academic, New York, 1991).

[5] K. B. Efetov, Adv. Phys. 32, 53 (1983).

[6] B. L. Altshuler and B. I. Shklovskiř, Zh. Eksp. Teor. Fiz. 91, 220 (1986) [Sov. Phys. JETP 64, 127 (1986)].

[7] A. Szafer and B. L. Altshuler, Phys. Rev. Lett. 70, 587 (1993).

[8] B. D. Simons and B. L. Altshuler (to be published).

[9] F. J. Dyson, J. Math. Phys. 3, 1191 (1962); 13, 90 (1972).

[10] G. Lenz and F. Haake, Phys. Rev. Lett. 65, 2325 (1990); F. Haake, Quantum Signatures of Chaos (Springer, Berlin, 1992). Earlier, related references are: $H$. Hasegawa, H. J. Mikeska, and H. Frahm, Phys. Rev. A 38, 395 (1988); M. Wilkinson, J. Phys. A 21, 1173 (1988); F. Leyvraz and T. H. Seligman, J. Phys. A 23, 1555 (1990).

[11] C. W. J. Beenakker, Phys. Rev. Lett. 70, 1155 (1993); Phys. Rev. B 47, 15763 (1993).

[12] E. Akkermans and G. Montambaux, Phys. Rev. Lett. 68, 642 (1992).

[13] K. A. Muttalib, J.-L. Pichard, and A. D. Stone, Phys. Rev. Lett. 59, 2475 (1987).

[14] B. D. Simons, P. A. Lee, and B. L. Altshuler, preceding Letter, Phys. Rev. Lett. 70, • • (1993).

[15] B. Sutherland, Phys. Rev. A 5, 1372 (1972). 